\title{
Phenological changes and reduced seasonal synchrony in western Poland
}

\author{
Tim H. Sparks • Maria Górska-Zajączkowska • \\ Wanda Wójtowicz • Piotr Tryjanowski
}

Received: 15 March 2010 /Revised: 1 July 2010 / Accepted: 28 July 2010/Published online: 29 August 2010

(C) The Author(s) 2010. This article is published with open access at Springerlink.com

\begin{abstract}
Botanical gardens offer continuity for phenological recording in observers, protocols and plant specimens that may not be achievable from other sources. Here, we examine phenological change and synchrony from one such garden in western Poland. We analysed 66 botanical phenophases and 18 interphase intervals recorded between 1977 and 2007 from the Poznań Botanical Garden. These were examined for trends through time and responsiveness to temperature. Furthermore, we derived measures of synchrony for start of spring and end of autumn events to assess if these had changed over time. All 39 events with a mean date before mid-July demonstrated a significant negative relationship with temperature. Where autumn events were significantly related to temperature, they indicated a positive relationship. Typically, spring events showed an advance over time and autumn events a delay. Interphase intervals tended to lengthen over the study period. The measures of synchrony changed significantly over time suggesting less synchrony among spring events and also among autumn events. In combination, these results suggest increases in growing season length. However, responses to a changing climate were species-specific. Thus, the transitions from winter into spring and from autumn into winter are becoming less clearly defined.
\end{abstract}

Keywords Botanical garden · Fall (autumn) - Flowering · Leafing $\cdot$ Temperature response $\cdot$ Trends

\section{T. H. Sparks $(\bowtie) \cdot$ P. Tryjanowski}

Institute of Zoology, Poznań University of Life Sciences,

Wojska Polskiego $71 \mathrm{C}$,

60-625 Poznań, Poland

e-mail: thsparks@btopenworld.com

M. Górska-Zajączkowska • W. Wójtowicz

Botanical Garden, Adam Mickiewicz University,

Dąbrowskiego 165,

60-594 Poznań, Poland

\section{Introduction}

Phenology is one of the most sensitive responses of the natural world to a changing climate. Of all the evidence considered by the IPCC in its most recent report, the bulk of evidence of changes to the natural world concerned phenological change (Rosenzweig et al. 2008). Indeed, phenological change may be seen as a vanguard for wider change in the environment (Cleland et al. 2007).

Plant phenology has been shown to be very responsive to temperatures, and a number of multi-species studies have reported shifting phenology, particularly to earlier leafing and flowering in spring (Bradley et al. 1999; Menzel and Fabian 1999; Abu-Asab et al. 2001; Fitter and Fitter 2002; Peñuelas et al. 2002; Menzel et al. 2006). However, many of these papers covered data only up to the end of the twentieth century and had a focus on spring events. Reports on autumn phenology are much less common (but see Menzel and Fabian 1999), and there have been few multispecies papers covering the first few years of the twentyfirst century which, because of their exceptional warmth, would be expected to be associated with exceptionally early spring phenology (e.g. White et al. 2009).

Studies that report many species have distinct advantages. They allow a comparison between species without the confounding environmental differences associated with different studies. Studies of records from a limited geographical area have further benefits in that the genetic diversity of the recorded material is likely to be smaller and the studied environment likely more homogenous (e.g. Hepper 2003). In this respect, botanical gardens may be particularly valuable in recording phenology. They are often long-established with their own meteorological station and continuity of personnel. Careful observation of various phases of the same species may well be possible 
without the need to search the environment to locate a particular species.

There have been few studies that have looked at several phases of the same species and the relationships between successive phases. Furthermore, there have been few multi-species studies from Eastern Europe (see maps in: Rosenzweig et al. 2008).

In this paper, we examine a 31-year record (1977-2007) of 66 phenophases from the Poznań Botanical Garden (Poland). The studied species include a number of iconic and more obscure ones. Of the former, Horse Chestnut Aesculus hippocastanum has been widely planted in Europe, has very obvious phenological phases, and has been widely reported in the phenological literature, for example the two-century record of first leafing from Geneva, Switzerland (Defila and Clot 2001). The purpose of our paper is to (1) identify trends in plant phenology from early spring to late autumn, (2) estimate the responsiveness of species to mean monthly air temperatures, (3) investigate the interphase intervals of the same species, and (4) look at the consistency of changes at the beginning and end of the growing season.

\section{Materials and methods}

A large number of plant phenophases have been monitored at the Adam Mickiewicz University Botanical Garden in Poznań, Poland (www.ogrod.edu.pl/info_eng. php) $\left(52^{\circ} 25^{\prime} \mathrm{N} 16^{\circ} 53^{\prime} \mathrm{E}\right)$. The Botanical Garden was founded in 1925, occupies an area of 22 ha at an altitude of $89 \mathrm{~m}$ asl and contains nearly 7,000 Special Collections. For this paper, we abstracted data on the dates of 66 phenophases for the period 1977-2007 incorporating 42 species (see Table 1 for list). All observations were made within the Garden and on a daily basis. The definitions of the phenophases used are as follows:First shoot - first shoots appearing above the ground; First leaf - first fully open leaf; First flower - first open flower; Pollen - first pollen shed; End of flowering - last flower; Earing inflorescence of cereals emerges; Seeding - first ripe seeds produced; First senescence - first evidence of above ground portion of plants dying; Leaf colouring - first colour change; Die back - above ground portions of plant fully dead; Bare - all leaves fallen.

All dates were converted prior to analysis into days after 31 December, hereafter day of the year (DOY) where $1=1$ January,etc. Eighteen intervals between successive phenophases of the same species were calculated where considered biologically meaningful (see Table 2 for list).

Mean monthly air temperatures, collected to standard WMO guidelines, were obtained from the meteorological station situated within the Botanical Garden.
Trends through time were estimated using linear regression of phenophases on year. Temperature responses were estimated by regression of phenophases on the mean temperature for the three calendar months ending in the month in which the mean of the phenophase occurred; thus, for example, an event whose mean date was in May would be compared to the mean temperature from March to May. This is a rather broadbrush approach but has been shown to be usually sufficient, particularly for spring events (Estrella et al. 2007).

The 18 phenophase intervals were subjected to regression on year to check for trends over time. A correlation was calculated between the two phases from which the interval was derived. Finally the end phases were regressed on the first phases after fitting the 3-month mean temperature mentioned above. This was in order to see if the first phases influenced the later ones after temperature effects were removed.

To assess variability changes within early spring events we calculated the standard deviation annually among all six phenophases occurring, on average, before the end of March. These are the first six events in Table 1. A similar exercise to look at autumn variability was based on the standard deviation of the eight "bare" phenophases listed towards the end of Table 1 . Trends in these variability measures were assessed by correlation with year.

\section{Results}

Trends through time

Table 1 summarises the examined phenophases, their trends through time and their response to the mean temperature of the three calendar months leading up to and including the mean date of that phase. Significant changes in timing were detected in 22 of the 66 phenophases; 14 significant advances and 8 significant delays. There was a strong association between timing of the phase and the trend through time $\left(r_{64}=0.624, P<0.001\right.$; Fig. 1$)$ with spring events tending to get earlier and autumn events later.

\section{Response to temperature}

In comparison with 3-monthly mean temperatures, 44 of the 66 phenophases showed a significant response to temperature (Table 1). Of these, 39 indicated earlier events with warmer temperatures. All events with mean dates before DOY 193 (July 12) had a significant negative relationship (warmer $=$ earlier) with temperature. The five significantly positive relationships all occurred in events with mean dates post DOY 274 (October 1). Overall, a strong correlation between temperature response and mean date was also apparent $\left(r_{64}=0.825, P<0.001\right.$; Fig. 2$)$. 
Table 1 A summary of the examined phenophases and the regressions of phenophases on year and on mean temperature

\begin{tabular}{|c|c|c|c|c|c|c|c|c|c|c|c|c|c|}
\hline \multirow[t]{2}{*}{ Scientific name } & \multirow[t]{2}{*}{ English name } & \multirow[t]{2}{*}{ Phase } & \multirow[t]{2}{*}{$\begin{array}{l}\text { Mean } \\
\text { (DOY) }\end{array}$} & \multirow[t]{2}{*}{ SD } & \multirow[t]{2}{*}{$n$} & \multicolumn{4}{|c|}{ Regression on year } & \multicolumn{4}{|c|}{$\begin{array}{l}\text { Regression on } 3 \text { months } \\
\text { temperature }\end{array}$} \\
\hline & & & & & & $\begin{array}{l}\text { slope } \\
\text { days/year }\end{array}$ & $\mathrm{SE}$ & $P$ & $R^{2}$ & $\begin{array}{l}\text { slope } \\
\text { days } /{ }^{\circ} \mathrm{C}\end{array}$ & $\mathrm{SE}$ & $P$ & $R^{2}$ \\
\hline Corylus avellana & Hazel & Pollen & 56.5 & 24.9 & 31 & -0.83 & 0.48 & 0.097 & 9.2 & -8.94 & 1.31 & $<0.001$ & 61.4 \\
\hline Pulmonaria obscura & Suffolk Lungwort & First shoot & 58.3 & 23.7 & 29 & -0.28 & 0.49 & 0.572 & 1.2 & -8.60 & 1.40 & $<0.001$ & 58.3 \\
\hline Alnus incana & Grey Alder & Pollen & 60.0 & 22.6 & 31 & -0.81 & 0.44 & 0.074 & 10.6 & -7.95 & 1.23 & $<0.001$ & 59.2 \\
\hline Galanthus nivalis & Snowdrop & First flower & 61.5 & 16.0 & 31 & -0.62 & 0.31 & 0.051 & 12.5 & -5.62 & 0.88 & $<0.001$ & $\mathbf{5 8 . 7}$ \\
\hline Leucojum vernum & Spring Snowflake & First shoot & 62.9 & 16.3 & 30 & -0.62 & 0.32 & 0.061 & 12.0 & -5.42 & 0.94 & $<0.001$ & 54.5 \\
\hline Lysimachia punctata & Dotted Loosestrife & First shoot & 63.1 & 21.1 & 31 & -0.76 & 0.41 & 0.072 & 10.8 & -7.27 & 1.18 & $<0.001$ & 56.7 \\
\hline Corylus avellana & Hazel & First leaf & 102.7 & 11.3 & 31 & -0.54 & 0.21 & 0.014 & 19.3 & -5.18 & 0.97 & $<0.001$ & 49.5 \\
\hline Convallaria majalis & Lily of the Valley & First shoot & 104.7 & 9.0 & 31 & -0.36 & 0.17 & 0.046 & 13.0 & -3.45 & 0.88 & $<0.001$ & 34.5 \\
\hline $\begin{array}{l}\text { Aesculus } \\
\text { hippocastanum }\end{array}$ & Horse Chestnut & First leaf & 104.8 & 7.8 & 31 & -0.31 & 0.15 & 0.044 & 13.3 & -2.94 & 0.78 & $<0.001$ & 33.0 \\
\hline Primula veris & Cowslip & First flower & 105.6 & 12.7 & 30 & -0.35 & 0.27 & 0.197 & 5.9 & -4.59 & 1.28 & $<0.001$ & 31.5 \\
\hline Larix decidua & European Larch & First leaf & 105.7 & 9.4 & 31 & -0.31 & 0.18 & 0.105 & 8.8 & -3.81 & 0.89 & $<0.001$ & 38.7 \\
\hline Cimicifuga europaea & Bugbane & First leaf & 108.5 & 8.5 & 24 & 0.47 & 0.23 & 0.056 & 15.6 & -2.39 & 0.98 & 0.023 & 21.3 \\
\hline Betula pendula & Silver Birch & First leaf & 108.9 & 9.2 & 31 & -0.08 & 0.19 & 0.659 & 0.7 & -2.72 & 0.99 & 0.010 & 20.8 \\
\hline Caltha palustris & Marsh Marigold & First flower & 110.1 & 9.5 & 31 & -0.40 & 0.18 & 0.033 & 14.7 & -3.57 & 0.94 & $<0.001$ & 33.2 \\
\hline Aristolochia clematitis & Birthwort & First shoot & 110.3 & 9.4 & 31 & -0.18 & 0.19 & 0.343 & 3.1 & -3.17 & 0.97 & 0.003 & 26.9 \\
\hline $\begin{array}{l}\text { Polygonatum } \\
\text { multiflorum }\end{array}$ & Solomon's Seal & First leaf & 111.1 & 8.8 & 30 & -0.32 & 0.17 & 0.069 & 11.3 & -3.86 & 0.83 & $<0.001$ & 43.5 \\
\hline Fritillaria imperialis & Crown Imperial & First flower & 111.2 & 7.9 & 30 & -0.22 & 0.16 & 0.163 & 6.8 & -3.23 & 0.78 & $<0.001$ & 37.9 \\
\hline Syringa vulgaris & Lilac & First flower & 126.1 & 7.6 & 31 & -0.44 & 0.13 & 0.002 & 27.7 & -4.56 & 0.89 & $<0.001$ & 47.5 \\
\hline $\begin{array}{l}\text { Aesculus } \\
\text { hippocastanum }\end{array}$ & Horse Chestnut & First flower & 126.4 & 7.1 & 31 & -0.50 & 0.11 & $<0.001$ & 40.1 & -5.06 & 0.67 & $<0.001$ & 66.4 \\
\hline Taraxacum officinale & Dandelion & Seeding & 129.3 & 7.6 & 31 & -0.52 & 0.12 & $<0.001$ & 38.9 & -5.26 & 0.73 & $<0.001$ & 63.9 \\
\hline $\begin{array}{r}\text { Polygonatum } \\
\text { multiflorum }\end{array}$ & Solomon's Seal & First flower & 130.6 & 9.4 & 31 & -0.68 & 0.14 & $<0.001$ & 43.2 & -5.70 & 1.08 & $<0.001$ & 49.1 \\
\hline Primula veris & Cowslip & $\begin{array}{l}\text { End of } \\
\text { flowering }\end{array}$ & 135.1 & 8.2 & 31 & -0.22 & 0.16 & 0.185 & 6.0 & -5.03 & 0.93 & $<0.001$ & 50.5 \\
\hline Secale cereale & Rye & Earing & 137.6 & 7.4 & 31 & -0.35 & 0.14 & 0.017 & 18.1 & -4.64 & 0.83 & $<0.001$ & 52.0 \\
\hline Caltha palustris & Marsh Marigold & $\begin{array}{l}\text { End of } \\
\text { flowering }\end{array}$ & 141.4 & 6.9 & 30 & -0.05 & 0.14 & 0.742 & 0.4 & -3.71 & 0.87 & $<0.001$ & 39.2 \\
\hline Robinia pseudoacacia & False Acacia & First flower & 146.6 & 9.4 & 31 & -0.35 & 0.18 & 0.067 & 11.1 & -6.33 & 0.96 & $<0.001$ & 60.0 \\
\hline Sambucus nigra & Elder & First flower & 148.1 & 10.0 & 31 & -0.36 & 0.19 & 0.073 & 10.7 & -7.91 & 0.65 & $<0.001$ & 83.7 \\
\hline Leucojum vernum & Spring Snowflake & $\begin{array}{l}\text { First } \\
\text { senescence }\end{array}$ & 151.5 & 10.7 & 30 & -0.41 & 0.22 & 0.068 & 11.4 & -7.17 & 1.08 & $<0.001$ & 61.1 \\
\hline $\begin{array}{l}\text { Physocarpus } \\
\text { opulifolius }\end{array}$ & Ninebark & First flower & 151.6 & 9.3 & 31 & 0.04 & 0.19 & 0.834 & 0.2 & -5.61 & 1.09 & $<0.001$ & 47.8 \\
\hline Fritillaria imperialis & Crown Imperial & $\begin{array}{l}\text { First } \\
\text { senescence }\end{array}$ & 154.8 & 12.0 & 31 & -0.28 & 0.24 & 0.259 & 4.4 & -5.55 & 1.80 & 0.004 & 24.7 \\
\hline Aruncus sylvestris & Bridewort & First flower & 154.9 & 7.1 & 31 & -0.18 & 0.14 & 0.212 & 5.3 & -4.25 & 0.95 & $<0.001$ & 40.6 \\
\hline Clematis recta & Erect Clematis & First flower & 155.5 & 7.8 & 31 & -0.23 & 0.15 & 0.138 & 7.4 & -4.87 & 1.00 & $<0.001$ & 44.9 \\
\hline Caltha palustris & Marsh Marigold & Seeding & 158.0 & 11.3 & 31 & 0.08 & 0.23 & 0.718 & 0.5 & -3.83 & 1.82 & 0.044 & 13.3 \\
\hline Cichorium intybus & Chicory & First flower & 176.1 & 11.0 & 31 & -0.48 & 0.21 & 0.026 & 15.9 & -6.39 & 1.49 & $<0.001$ & 38.9 \\
\hline Lilium martagon & Martagon Lily & $\begin{array}{l}\text { End of } \\
\text { flowering }\end{array}$ & 182.5 & 9.0 & 31 & -0.48 & 0.16 & 0.005 & 23.7 & -5.55 & 0.88 & $<0.001$ & $\mathbf{5 8 . 0}$ \\
\hline Lupinus polyphyllus & Garden Lupin & Seeding & 184.2 & 16.0 & 31 & -0.61 & 0.31 & 0.055 & 12.1 & -6.11 & 2.14 & 0.008 & 21.9 \\
\hline Hieracium umbellatum & $\begin{array}{l}\text { Umbellate } \\
\text { Hawkweed }\end{array}$ & First flower & 190.2 & 10.0 & 30 & 0.11 & 0.21 & 0.627 & 0.9 & -3.10 & 1.40 & 0.035 & 15.0 \\
\hline $\begin{array}{l}\text { Astragalus } \\
\text { glycyphyllos }\end{array}$ & Wild Liquorice & $\begin{array}{l}\text { End of } \\
\text { flowering }\end{array}$ & 190.8 & 12.4 & 29 & -0.61 & 0.24 & 0.019 & 18.8 & -6.75 & 1.47 & $<0.001$ & 44.0 \\
\hline Tilia cordata & Small-leaved Lime & $\begin{array}{l}\text { End of } \\
\text { flowering }\end{array}$ & 192.2 & 9.9 & 31 & -0.25 & 0.20 & 0.217 & 5.2 & -5.69 & 1.05 & $<0.001$ & 50.3 \\
\hline
\end{tabular}


Table 1 (continued)

\begin{tabular}{|c|c|c|c|c|c|c|c|c|c|c|c|c|c|}
\hline \multirow[t]{2}{*}{ Scientific name } & \multirow[t]{2}{*}{ English name } & \multirow[t]{2}{*}{ Phase } & \multirow[t]{2}{*}{$\begin{array}{l}\text { Mean } \\
\text { (DOY) }\end{array}$} & \multirow[t]{2}{*}{ SD } & \multirow[t]{2}{*}{$n$} & \multicolumn{4}{|c|}{ Regression on year } & \multicolumn{4}{|c|}{$\begin{array}{l}\text { Regression on } 3 \text { months } \\
\text { temperature }\end{array}$} \\
\hline & & & & & & $\begin{array}{l}\text { slope } \\
\text { days/year }\end{array}$ & SE & $P$ & $R^{2}$ & $\begin{array}{l}\text { slope } \\
\text { days } /{ }^{\circ} \mathrm{C}\end{array}$ & $\mathrm{SE}$ & $P$ & $R^{2}$ \\
\hline Lysimachia punctata & Dotted Loosestrife & $\begin{array}{l}\text { End of } \\
\text { flowering }\end{array}$ & 193.0 & 10.7 & 31 & -0.42 & 0.20 & 0.050 & 12.6 & -6.03 & 1.16 & $<0.001$ & 48.4 \\
\hline Solidago canadensis & Canadian Goldenrod & First flower & 205.6 & 14.8 & 31 & 1.15 & 0.21 & $<0.001$ & 50.3 & -0.25 & 2.23 & 0.913 & 0.0 \\
\hline Campanula trachelium & $\begin{array}{l}\text { Nettle-leaved } \\
\text { Bellflower }\end{array}$ & Seeding & 229.3 & 9.3 & 31 & -0.02 & 0.19 & 0.913 & 0.0 & -2.21 & 1.41 & 0.127 & 7.9 \\
\hline Sedum spectabile & Butterfly Stonecrop & First flower & 239.6 & 9.3 & 31 & 0.50 & 0.17 & 0.006 & 23.5 & 1.49 & 1.44 & 0.310 & 3.5 \\
\hline Lysimachia punctata & Dotted Loosestrife & Seeding & 242.5 & 11.7 & 31 & 0.42 & 0.22 & 0.070 & 10.9 & -1.75 & 1.81 & 0.340 & 3.1 \\
\hline Cimicifuga europaea & Bugbane & Seeding & 246.1 & 15.1 & 17 & -0.36 & 0.54 & 0.522 & 2.8 & -2.89 & 2.99 & 0.349 & 5.9 \\
\hline Solidago canadensis & Canadian Goldenrod & Seeding & 247.3 & 13.9 & 31 & 0.66 & 0.26 & 0.015 & 18.7 & 1.24 & 1.91 & 0.523 & 1.4 \\
\hline Sambucus nigra & Elder & Seeding & 251.0 & 12.4 & 30 & 0.14 & 0.26 & 0.613 & 0.9 & -1.82 & 1.75 & 0.307 & 3.7 \\
\hline $\begin{array}{l}\text { Aesculus } \\
\text { hippocastanum }\end{array}$ & Horse Chestnut & Seeding & 256.2 & 7.4 & 31 & -0.05 & 0.15 & 0.724 & 0.4 & -0.16 & 1.03 & 0.877 & 0.1 \\
\hline $\begin{array}{l}\text { Aesculus } \\
\text { hippocastanum }\end{array}$ & Horse Chestnut & Leaf colouring & 269.9 & 10.0 & 28 & -0.48 & 0.20 & 0.027 & 17.5 & -0.35 & 1.48 & 0.817 & 0.2 \\
\hline Betula pendula & Silver Birch & Leaf colouring & 274.5 & 10.9 & 31 & 0.21 & 0.22 & 0.358 & 2.9 & 4.82 & 2.01 & 0.023 & 16.5 \\
\hline Corylus avellana & Hazel & Leaf colouring & 276.6 & 12.5 & 30 & 0.20 & 0.26 & 0.444 & 2.1 & 4.96 & 2.35 & 0.043 & 13.8 \\
\hline Cimicifuga europaea & Bugbane & Die back & 286.6 & 11.4 & 19 & -0.04 & 0.35 & 0.912 & 0.1 & -1.42 & 2.94 & 0.636 & 1.4 \\
\hline Colchicum autumnale & Meadow Saffron & $\begin{array}{l}\text { End of } \\
\text { flowering }\end{array}$ & 287.1 & 10.6 & 31 & 0.44 & 0.20 & 0.037 & 14.2 & 0.03 & 2.13 & 0.987 & 0.0 \\
\hline $\begin{array}{l}\text { Vincetoxicum } \\
\text { hirundinaria }\end{array}$ & Swallow-wort & Die back & 291.5 & 13.5 & 31 & 0.13 & 0.27 & 0.637 & 0.8 & -3.19 & 2.64 & 0.238 & 4.8 \\
\hline Paeonia officinalis & Peony & Die back & 296.2 & 13.2 & 31 & 0.54 & 0.25 & 0.040 & 13.8 & 1.37 & 2.65 & 0.610 & 0.9 \\
\hline $\begin{array}{l}\text { Aesculus } \\
\text { hippocastanum }\end{array}$ & Horse Chestnut & Bare & 308.0 & 7.0 & 28 & 0.02 & 0.16 & 0.894 & 0.1 & 0.55 & 1.20 & 0.649 & 0.8 \\
\hline Acer platanoides & Norway Maple & Bare & 312.3 & 8.0 & 30 & 0.25 & 0.16 & 0.122 & 8.3 & 1.74 & 1.31 & 0.193 & 6.0 \\
\hline Phlox paniculata & Perennial Phlox & Die back & 312.8 & 9.4 & 31 & -0.23 & 0.19 & 0.229 & 4.9 & -0.23 & 1.58 & 0.884 & 0.1 \\
\hline Viburnum opulus & Guelder Rose & Bare & 313.3 & 8.4 & 31 & -0.12 & 0.17 & 0.499 & 1.6 & 0.56 & 1.41 & 0.696 & 0.5 \\
\hline Syringa vulgaris & Lilac & Bare & 314.1 & 8.2 & 31 & -0.03 & 0.17 & 0.870 & 0.1 & 2.18 & 1.33 & 0.112 & 8.5 \\
\hline Paeonia sinensis & Chinese Peony & Die back & 314.7 & 8.9 & 31 & 0.01 & 0.18 & 0.972 & 0.0 & 1.38 & 1.47 & 0.357 & 2.9 \\
\hline Iris sibirica & Siberian Iris & Die back & 314.9 & 10.7 & 31 & -0.13 & 0.22 & 0.552 & 1.2 & 3.76 & 1.66 & 0.031 & 15.1 \\
\hline Betula pendula & Silver Birch & Bare & 316.9 & 10.2 & 31 & 0.56 & 0.18 & 0.005 & 24.6 & 3.89 & 1.56 & 0.019 & 17.5 \\
\hline Tilia cordata & Small-leaved Lime & Bare & 317.8 & 9.8 & 31 & 0.55 & 0.17 & 0.003 & 25.9 & 3.80 & 1.49 & 0.017 & 18.2 \\
\hline Lysimachia punctata & Dotted Loosestrife & Die back & 319.2 & 10.6 & 31 & 0.08 & 0.22 & 0.709 & 0.5 & 1.70 & 1.76 & 0.342 & 3.1 \\
\hline Larix decidua & European Larch & Bare & 326.5 & 11.9 & 31 & 0.27 & 0.24 & 0.258 & 4.4 & 3.81 & 1.88 & 0.052 & 12.4 \\
\hline Salix fragilis & Crack Willow & Bare & 326.7 & 15.1 & 31 & 1.21 & 0.21 & $<0.001$ & 53.3 & 4.39 & 2.41 & 0.079 & 10.3 \\
\hline
\end{tabular}

Regressions in bold are statistically significant $P<0.05$, phenophases are arranged in order of mean date

DOY Day of year (days after 31 December)

A highly significant correlation existed between the regression estimates of phenophase on year and the regression estimates of phenophase on temperature, i.e. columns 7 and 11 in Table $1\left(r_{64}=0.741, P<0.001\right)$.

Trends and temperature responses in interphase intervals

Table 2 lists the 18 considered intervals. Seven of these had changed significantly during the study period; all of them getting longer. Fifteen of the 18 intervals had positive trends through time suggesting extended phase intervals for the majority of species, some of which can be interpreted loosely as the length of the growing season. For 7 of these intervals significant positive correlations existed between the two phases used to derive the interval (Table 2). However, the correlations were not typically large suggesting that the intervals are rarely predetermined, but rather influenced by annual climatic conditions. In fact only 3 of the earlier phases were significant in modelling the later phase once temperature effects had been accounted for (Table 2). 
Table 2 Trends in 18 phase intervals, the correlation between the two phases and the significance of the earlier phases in a regression model after fitting 3-month temperature

\begin{tabular}{|c|c|c|c|c|c|c|c|c|c|c|c|c|}
\hline \multirow[t]{2}{*}{ Scientific name } & \multirow[t]{2}{*}{ Phase } & \multirow[t]{2}{*}{$\begin{array}{l}\text { Earlier } \\
\text { phase }\end{array}$} & \multirow[t]{2}{*}{$\begin{array}{l}\text { Mean } \\
\text { (DOY) }\end{array}$} & \multirow[t]{2}{*}{ SD } & \multirow[t]{2}{*}{$n$} & \multicolumn{4}{|c|}{ Regression on year } & \multirow{2}{*}{$\begin{array}{l}\text { Correlation } \\
\text { between phases } \\
r\end{array}$} & \multicolumn{2}{|c|}{$\begin{array}{l}\text { Influence and } \\
\text { significance of } \\
\text { earlier phase after } \\
\text { fitting temperature }\end{array}$} \\
\hline & & & & & & $\begin{array}{l}\text { slope } \\
\text { days/year }\end{array}$ & $\mathrm{SE}$ & $P$ & $R^{2}$ & & $\begin{array}{l}\text { Slope } \\
\text { days/day }\end{array}$ & $P$ \\
\hline $\begin{array}{l}\text { Aesculus } \\
\text { hippocastanum }\end{array}$ & Seeding & First flower & 129.7 & 8.4 & 31 & 0.444 & 0.151 & 0.006 & 23.1 & 0.34 & 0.367 & 0.066 \\
\hline $\begin{array}{l}\text { Aesculus } \\
\text { hippocastanum }\end{array}$ & $\begin{array}{l}\text { Leaf } \\
\text { colouring }\end{array}$ & First leaf & 164.9 & 9.4 & 28 & -0.134 & 0.208 & 0.526 & 1.6 & 0.48 & 0.637 & 0.010 \\
\hline $\begin{array}{l}\text { Aesculus } \\
\text { hippocastanum }\end{array}$ & Bare & First leaf & 203.0 & 10.8 & 28 & 0.366 & 0.230 & 0.124 & 8.8 & -0.01 & -0.011 & 0.950 \\
\hline $\begin{array}{l}\text { Aesculus } \\
\text { hippocastanum }\end{array}$ & Bare & $\begin{array}{l}\text { Leaf } \\
\text { colouring }\end{array}$ & 38.1 & 11.9 & 28 & 0.500 & 0.248 & 0.055 & 13.5 & 0.06 & 0.035 & 0.805 \\
\hline Betula pendula & $\begin{array}{l}\text { Leaf } \\
\text { colouring }\end{array}$ & First leaf & 165.5 & 14.8 & 31 & 0.288 & 0.298 & 0.341 & 3.1 & -0.08 & -0.035 & 0.869 \\
\hline Betula pendula & Bare & First leaf & 208.0 & 11.6 & 31 & 0.640 & 0.204 & 0.004 & 25.4 & 0.29 & 0.329 & 0.086 \\
\hline Betula pendula & Bare & $\begin{array}{l}\text { Leaf } \\
\text { colouring }\end{array}$ & 42.5 & 12.6 & 31 & 0.352 & 0.250 & 0.169 & 6.4 & 0.29 & 0.153 & 0.370 \\
\hline Caltha palustris & Last flower & First flower & 31.7 & 7.6 & 30 & 0.321 & 0.146 & 0.037 & 14.7 & 0.59 & 0.233 & 0.107 \\
\hline Caltha palustris & Seeding & First flower & 48.0 & 13.2 & 31 & 0.485 & 0.253 & 0.066 & 11.2 & 0.20 & 0.103 & 0.645 \\
\hline Cimicifuga europaea & Die back & First leaf & 180.9 & 12.8 & 17 & -0.776 & 0.432 & 0.093 & 17.7 & 0.18 & 0.319 & 0.396 \\
\hline Corylus avellana & $\begin{array}{l}\text { Leaf } \\
\text { colouring }\end{array}$ & First leaf & 174.0 & 16.5 & 30 & 0.764 & 0.314 & 0.022 & 17.5 & 0.06 & 0.160 & 0.426 \\
\hline Larix decidua & Bare & First leaf & 220.7 & 14.4 & 31 & 0.581 & 0.273 & 0.042 & 13.5 & 0.11 & 0.141 & 0.532 \\
\hline Leucojum vernum & Senescence & First shoot & 88.4 & 15.3 & 29 & 0.294 & 0.333 & 0.385 & 2.8 & 0.44 & -0.019 & 0.845 \\
\hline Lysimachia punctata & Seeding & Last flower & 49.5 & 14.1 & 31 & 0.840 & 0.242 & 0.002 & 29.3 & 0.20 & 0.166 & 0.496 \\
\hline Lysimachia punctata & Die back & First shoot & 256.1 & 19.9 & 31 & 0.842 & 0.376 & 0.033 & 14.7 & 0.36 & 0.176 & 0.055 \\
\hline Primula veris & Last flower & First flower & 29.5 & 11.9 & 30 & 0.107 & 0.255 & 0.676 & 0.6 & 0.42 & 0.014 & 0.899 \\
\hline Sambucus nigra & Seeding & First flower & 103.0 & 12.4 & 30 & 0.501 & 0.247 & 0.053 & 12.8 & 0.41 & 0.475 & 0.039 \\
\hline Solidago canadensis & Seeding & First flower & 41.7 & 14.2 & 31 & -0.493 & 0.275 & 0.083 & 10.0 & 0.51 & 0.480 & 0.005 \\
\hline
\end{tabular}

Results in bold are statistically significant $P<0.05$

DOY Day of year (days after 31 December)

Inter-species variability in spring and autumn

The standard deviation between the six early phenophases plotted against year is shown in Fig. 3. This has significantly increased over time $\left(r_{29}=0.520, P=0.003\right)$ and is greater in early springs (correlation with the mean date of the six phenophases $\left.r_{29}=-0.502, P=0.004\right)$. The standard deviation between the eight "bare" phenophases plotted against year is shown in Fig. 4. This has also significantly increased over time $\left(r_{29}=0.553, P=0.001\right)$ and is greater in late autumns (correlation with the mean date of the eight phenophases $\left.r_{29}=0.720, P<0.001\right)$.

\section{Discussion}

Botanical gardens can offer many advantages in studies of phenology and climate impacts (Donaldson 2009; Primack and Miller-Rushing 2009). They are typically long established, with professional staff and good archives. There is typically a stability in both staffing and methods that results in continuity of recording protocols, and a longevity that can rarely be achieved when records are made by individuals (but see Fitter and Fitter 2002). Phenological recording may often involve the same specimen in a relatively small area and thus eliminate some of the noise associated with phenological records made in the wild over large areas. Their compact area also makes interspecies comparisons more valid since environmental conditions will be much more similar. Many botanical gardens, such as that in Poznań, also have their own meteorological station enhancing the value of the plant records that have been made. Botanical garden archives offer additional possibilities (Miller-Rushing and Primack 2008; Donaldson 2009; Primack and Miller-Rushing 2009). Sadly, we are not aware of any other contemporary species-rich data sources within Poland with which to compare our data. 


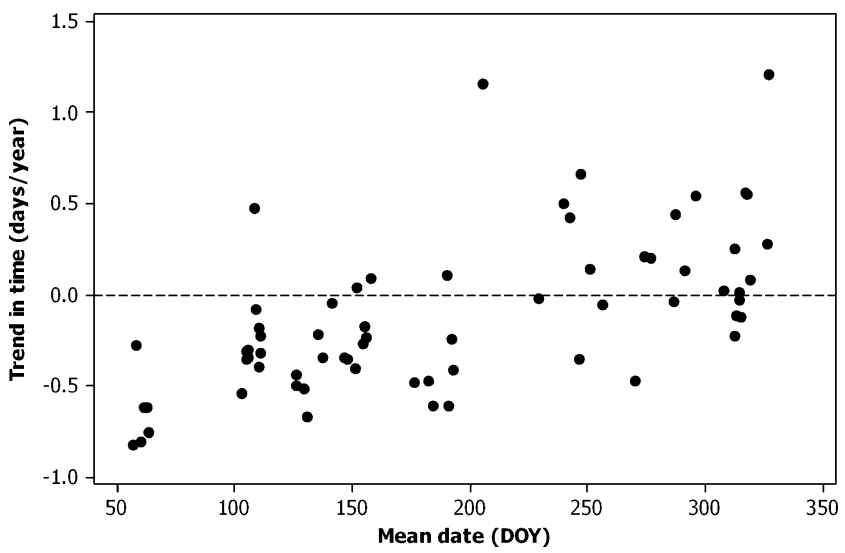

Fig. 1 Trends through time (days/year) for 66 phenophases recorded at the Poznań Botanical Garden in the period 1977-2007 plotted against the mean date (day of the year) of the phenophase. A dotted reference line has been added; phases below the line got earlier, above the line later

The results reported here confirm the responsiveness of plant phenology, particularly of spring events, to temperature. These help to confirm the value of plant phenology as a climate change indicator. Autumn events have typically been equivocal in their response to temperature but our results suggest a delay in autumn events associated with rising temperatures. Further work is needed to tease apart the relative importance of mean temperature and other autumn drivers such as wind, sunshine and frost on leaf fall. We investigated 18 interphase intervals. Seven of these had become significantly longer which broadly suggests a lengthening of the growing season. In all but three cases the earlier phase timing was not significant after temperature had been accounted for. Thus it appears that the later phases are far more influenced by prevailing temperature than the timing of preceding phases. However, for Aesculus

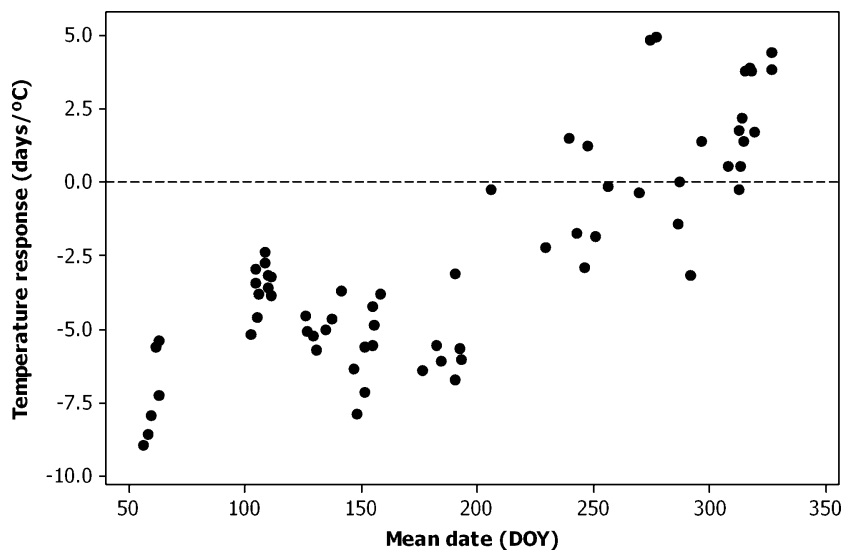

Fig. 2 Temperature responses $\left(\right.$ days $/{ }^{\circ} \mathrm{C}$ ) for 66 phenophases recorded at the Poznań Botanical Garden in the period 1977-2007 plotted against the mean date (day of the year) of the phenophase. A dotted reference line has been added; phases below the line getting earlier with warmer temperatures, above the line later

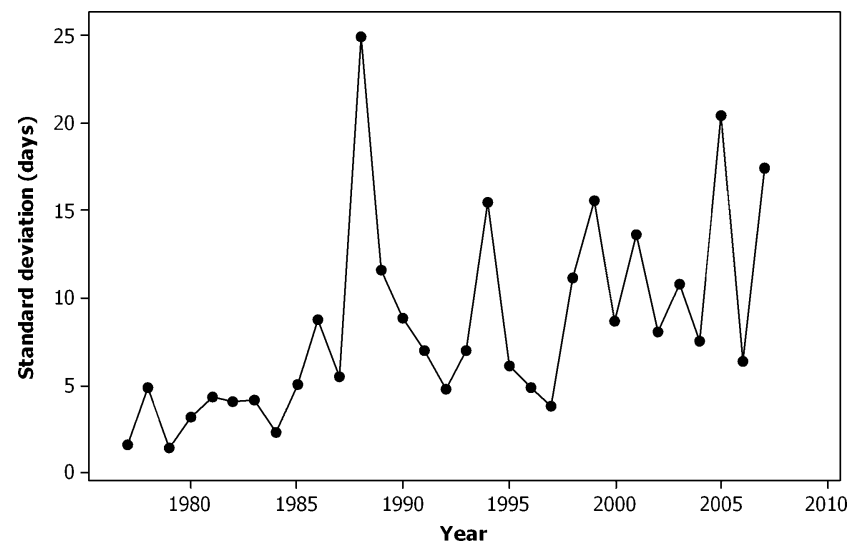

Fig. 3 The standard deviation between the dates of six early phenophases recorded at the Poznan Botanical Garden for the period 1977-2007

hippocastanum, leaf colouring appeared positively correlated with earlier phases suggesting that early leafing resulted in an earlier end of season. However, this pattern was not apparent in Betula pendula or Corylus avellana. Further investigation of whether leaves have a limited lifetime ("shelf life"), thus associating early springs with early autumns, may be justified (see also Cleland et al. 2007).

We calculated a measure of spring synchrony based on the standard deviation between the dates of the six early spring events, and a similar one for autumn based on the standard deviation between the bare dates for eight tree species. Both of these measures increased significantly over time indicating reduced synchrony in both seasons. Whilst we are limited in the choice of phenophases to assess synchrony, examination of their coefficients for trend and temperature response in Table 1 do not suggest that synchrony measures are overly influenced by a single phenophase. We believe that most people associate the seasons, particularly spring and autumn, with biological events. These results suggest that the sharply

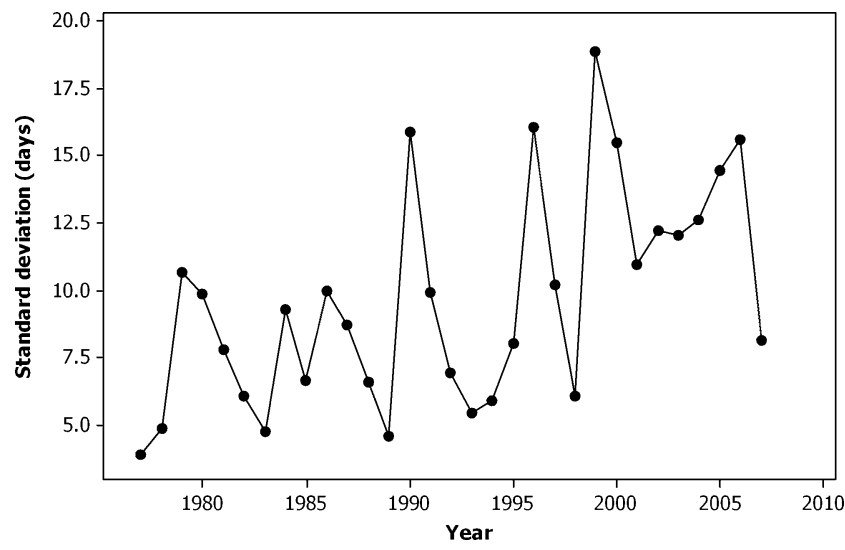

Fig. 4 The standard deviation between the dates of eight "bare" phenophases recorded at the Poznań Botanical Garden for the period 1977-2007 
defined spring at this mid-continent location at the beginning of our study period (low standard deviation in Fig. 3) has become less consistent over time. A similar change has occurred in autumn. Thus the perceived boundaries between winter and spring, and between autumn and winter, have become increasingly blurred. The consequences of this phenomenon to wildlife, particularly those with specific dependent links in food webs, remains to be seen.

Acknowledgements The authors thank MetOffice Poznań - Ławica staff for assistance with obtaining temperature data and David Inouye and two anonymous reviewers for comments on an earlier version of this paper.

Open Access This article is distributed under the terms of the Creative Commons Attribution Noncommercial License which permits any noncommercial use, distribution, and reproduction in any medium, provided the original author(s) and source are credited.

\section{References}

Abu-Asab MS, Peterson PM, Shetler SG, Orli SS (2001) Earlier plant flowering in spring as a response to global warming in the Washington, DC, area. Biodivers Conserv 10:597-612

Bradley NL, Leopold AC, Ross J, Huffaker W (1999) Phenological changes reflect climate change in Wisconsin. Proc Natl Acad Sci 96:9701-9704

Cleland EE, Chuine I, Menzel A, Mooney HA, Schwartz MD (2007) Shifting plant phenology in response to global change. Trends Ecol Evol 22:357-365
Defila C, Clot B (2001) Phytophenological trends in Switzerland. Int J Biometeorol 45:203-207

Donaldson JS (2009) Botanic gardens science for conservation and global change. Trends Plant Sci 14:608-613

Estrella N, Sparks TH, Menzel A (2007) Trends and temperature response in the phenology of crops in Germany. Glob Chang Biol 13:1737-1747

Fitter AH, Fitter RSR (2002) Rapid changes in flowering time in British plants. Science 296:1689-1691

Hepper FN (2003) Phenological records of English garden plants in Leeds (Yorkshire) and Richmond (Surrey) from 1946 to 2002. An analysis relating to global warming. Biodivers Conserv 12:2503-2520

Menzel A, Fabian P (1999) Growing season extended in Europe. Nature 397:659

Menzel A, Sparks TH, Estrella N, Koch E, Aasa A, Ahas R, AlmKübler K, Bissolli P, Braslavská O, Briede A et al (2006) European phenological response to climate change matches the warming pattern. Glob Chang Biol 12:1969-1976

Miller-Rushing AJ, Primack RB (2008) Global warming and flowering times in Thoreau's Concord: a community perspective. Ecology 89:332-341

Peñuelas J, Filella I, Comas P (2002) Changed plant and animal life cycles from 1952 to 2000 in the Mediterranean region. Glob Chang Biol 8:531-544

Primack RB, Miller-Rushing AJ (2009) The role of botanical gardens in climate change research. New Phytol 182:303-313

Rosenzweig C, Karoly D, Vicarelli M, Neofotis P, Wu Q, Cassara G, Menzel A, Root TL, Estrella N, Seguin B et al (2008) Attributing physical and biological impacts to anthropogenic climate change. Nature 453:353-358

White MA, de Beurs KM, Didan K, Inouye DW, Richardson AD, Jensen OP, O'Keefe J, Zhang G, Nemani RR, van Leuwen WJD et al (2009) Intercomparison, interpretation, and assessment of spring phenology in North America estimated from remote sensing for 1982-2006. Glob Chang Biol 15:2335-2359 